\title{
Disconnected Cuts in Claw-free Graphs
}

\author{
Barnaby Martin \\ Department of Computer Science, Durham University, Durham, UK \\ barnaby.d.martin@durham.ac.uk

\section{Daniël Paulusma} \\ Department of Computer Science, Durham University, Durham, UK \\ daniel.paulusma@durham.ac.uk
}

\section{Erik Jan van Leeuwen}

Department of Information and Computing Sciences, Utrecht University, The Netherlands e.j.vanleeuwen@uu.nl

\begin{abstract}
A disconnected cut of a connected graph is a vertex cut that itself also induces a disconnected subgraph. The corresponding decision problem is called DisconneCTED CuT. It is known that Disconnected Cut is NP-hard on general graphs, while polynomial-time algorithms exist for several graph classes. However, the complexity of the problem on claw-free graphs remained an open question. Its connection to the complexity of the problem to contract a claw-free graph to the 4 -vertex cycle $C_{4}$ led Ito et al. (TCS 2011) to explicitly ask to resolve this open question. We prove that DisCONNECTED CUT is polynomial-time solvable on claw-free graphs, answering the question of Ito et al. The basis for our result is a decomposition theorem for claw-free graphs of diameter 2, which we believe is of independent interest and builds on the research line initiated by Chudnovsky and Seymour (JCTB 2007-2012) and Hermelin et al. (ICALP 2011). On our way to exploit this decomposition theorem, we characterize how disconnected cuts interact with certain cobipartite subgraphs, and prove two further algorithmic results, namely that DisCONNECTED CUT is polynomial-time solvable on circular-arc graphs and line graphs.
\end{abstract}

2012 ACM Subject Classification Mathematics of computing $\rightarrow$ Graph theory

Keywords and phrases disconnected cut, surjective homomorphism, biclique cover, claw-freeness

Digital Object Identifier 10.4230/LIPIcs.ESA.2018.61

Related Version A full version is available at https://arxiv.org/abs/1803.03663.

Funding This paper received support from the Leverhulme Trust (RPG-2016-258).

\section{Introduction}

Graph connectivity is a crucial graph property studied in the context of network robustness. Well-studied notions of connectivity consider for example hamiltonicity, edge-disjoint spanning trees, edge cuts, vertex cuts, etc. In this paper, we study the notion of a disconnected cut, which is a vertex set $U$ of a connected graph $G$ such that $G-U$ is disconnected and the subgraph $G[U]$ induced by $U$ is disconnected as well. Alternatively, we say that $V(G)$ can be partitioned into nonempty sets $V_{1}, V_{2}, V_{3}, V_{4}$ such that no vertex of $V_{1}$ is adjacent to a vertex of $V_{3}$ (that is, $V_{1}$ is anti-complete to $V_{3}$ ) and $V_{2}$ is anti-complete to $V_{4}$; then both $V_{1} \cup V_{3}$ and $V_{2} \cup V_{4}$ form a disconnected cut. See Figure 1 for an example. The DisconneCted CuT problem asks whether a given connected graph $G$ has a disconnected cut.

cc) (i) Barnaby Martin, Daniël Paulusma, and Erik Jan van Leeuwen;

26th Annual Eicensed under Creative Commons License CC-BY

Editors: Yossi Azar, Hannah Bast, and Grzegorz Herman; Article No. 61; pp. 61:1-61:14

Leibniz International Proceedings in Informatics

LIPICS Schloss Dagstuhl - Leibniz-Zentrum für Informatik, Dagstuhl Publishing, Germany 


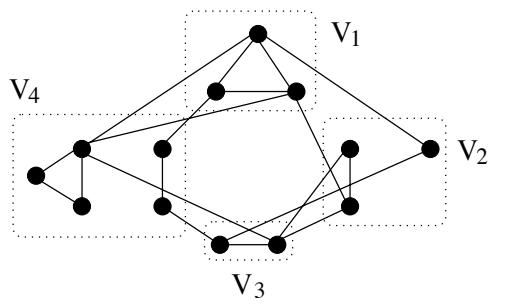

Figure 1 Graph with disconnected cuts $V_{1} \cup V_{3}$ and $V_{2} \cup V_{4}$ (figure originally appeared in [22]).

The Disconnected Cut problem is intimately connected to at least five other problems studied in the literature. We give a brief overview here, and refer to the related work section for more details. The name Disconnected CuT originates from Fleischner et al. [15], who determined the complexity of partitioning the vertices of a graph into exactly $k$ bicliques (complete bipartite graphs with at least one edge), except for the case $k=2$. For $k=2$, this problem is polynomially equivalent to DisconneCTED CUT (by taking the complement of the input graph). The Disconnected Cut problem can also be seen as an $H$-PARTition problem for appropriately defined 4-vertex graphs $H$. Dantas et al. [8] proved that $H$-PARTITION is polynomial-time solvable for each 4-vertex graph $H$ except for the two cases equivalent to Disconnected Cut. If the input graph has diameter 2, then Disconnected Cut is equivalent to $\mathcal{C}_{4}$-COMPACTION [15], which asks for a homomorphism $f$ from a graph $G$ to the graph $\mathcal{C}_{4}$ (the 4-vertex-cycle with a self-loop in each vertex) such that for every $x y \in E(H)$ with $x \neq y$ there is an edge $u v \in E(G)$ with $f(u)=x$ and $f(v)=y$. The diameter- 2 case is also equivalent to testing if a graph can be modified to a biclique by a series of edge contractions [22]. The restriction to graphs of diameter 2 is natural, as every graph of diameter 1 has no disconnected and every graph of diameter at least 3 has a disconnected cut [15]. Finally, DisconneCTED Cut fits in the broad study of vertex cut problems with extra properties on the cut set; see [23] for an overview.

The above demonstrates that Disconnected CUT is of central importance to understanding many different types of problems, ranging from cut problems to homomorphism and graph contractibility problems. Therefore, there has been broad interest to determine its computational complexity. Indeed, numerous papers $[6,8,9,10,15,21,22,28]$ asked about its complexity on general graphs. NP-completeness was proven independently in [26] and by Vikas, as announced in [30]. The strong interest in DisconneCted Cut also led to a study on graph classes. We know polynomial-time algorithms for many classes $[6,9,15,22]$, particularly for certain classes of $H$-free graphs (graphs without a fixed graph $H$ as an induced subgraph). However, even for several simple graphs $H$, the complexity landscape of DisconneCted Cut still contains gaps. Indeed, even for four-vertex graphs $H$, we show (in the full version of our paper) that one open case remains, namely the case $H=K_{4}$. To prove this result, we need to deal with one non-trivial case, namely, when $H=K_{1,3}$ (the claw).

Our interest in Disconnected Cut on claw-free graphs is heightened by the close relation of this problem to $C_{r}$-CONTRACTIBILITy, which is to decide if a graph $G$ contains the $r$-vertex cycle $C_{r}$ as a contraction. This problem is NP-complete if $r \geq 4$ [3] and stays NP-complete for claw-free graphs as long as $r \geq 6$ [14]. The case $r \leq 3$ is polynomial-time solvable even for general graphs [3]. Hence, for claw-free graphs this leaves open the cases where $r \in\{4,5\}$. Ito et al. [22] showed that $C_{4}$-CONTRACTIBILITY on claw-free graphs of diameter 2 is equivalent to Disconnected Cut. As Disconnected Cut is trivial if the input graph does not have diameter 2, this led Ito et al. [22] to explicitly ask the following:

What is the computational complexity of DiscONNECTED CUT on claw-free graphs? 
Our Contribution. We answer the open question of Ito et al. [22] by giving a polynomialtime algorithm for DisconneCTED Cut on claw-free graphs (which also finds a disconnected cut if it exists). This immediately implies that $\mathcal{C}_{4}$-Compaction and $C_{4}$-Contractibility are polynomial-solvable on claw-free graphs of diameter 2. As claw-free graphs are not closed under edge contraction, the latter is certainly not expected beforehand.

We start with the basic observation that Disconnected Cut is trivial if the graph does not have diameter 2 [15]. Hence, we aim for a deeper understanding of claw-free graphs of diameter 2. To this end, we give a new graph-theoretic theorem that proves that claw-free graphs of diameter 2 belong to one of four basic graph classes after performing two types of elementary operations. The theorem builds on one of the algorithmic decomposition theorems for claw-free graphs developed by Hermelin et al. [19, 20], and relies on the pioneering works of Chudnovsky and Seymour [5]. Several other algorithmic decomposition theorems for claw-free graphs have been built on the ideas of Chudnovsky and Seymour, see e.g. [11, 24], which jointly have had a broad impact on our algorithmic understanding of claw-free graphs (see [19] for an overview or [2]). Our structural theorem and resulting algorithm for DisconNECTED Cut expand this line of research.

The crux of the proof of our structural theorem is to exploit the extra structure offered by claw-free graphs of diameter 2 to show that the so-called strip-structures, which are central to the aforementioned decomposition theorems, only contain trivial strips. An important ingredient in the proof is to exclude not only twins (vertices $u, v$ for which $N[u]=N[v]$ ), but also vertices with nested neighbourhoods (vertices $u$ for which there exists a vertex $v$ such that $N(u) \backslash\{v\} \subseteq N(v) \backslash\{u\})$. Using this operation, one can simplify the decomposition theorem of [19], and we think this observation may have an impact beyond this work.

Using the structural theorem, Disconnected Cut on claw-free graphs reduces to understanding its behavior under the elementary operations and on the basic graph classes. The crucial elementary operation is to remove certain cobipartite structures known as Wjoins [5]. We develop the notion of unshatterable proper $\mathrm{W}$-joins, which are essentially $\mathrm{W}$-joins that cannot be broken into smaller W-joins, and exhibit how unshatterable proper $\mathrm{W}$-joins interact with disconnected cuts. We then show that unshatterable proper W-joins can be removed from the graph by a simple operation. We complete our arguments by proving that all $\mathrm{W}$-joins in the graph must be in fact be unshatterable proper $\mathrm{W}$-joins, and that we can find unshatterable proper $\mathrm{W}$-joins in polynomial time.

The main basic graph classes in the structural theorem are line graphs and proper circular-arc graphs. Prior to our work, the complexity of Disconnected CUT was unknown for these classes as well. We present a polynomial-time algorithm for line graphs and even for general circular-arcs graphs (not only proper circular-arcs). Both algorithms rely on the existence of a small induced cycle passing through a disconnected cut in a highly structured manner. In addition, for line graphs, we prove that the pre-image of the line graph is $2 P_{2}$-free, and thus has diameter at most 3. The hardest part of the proof is then to prove that if the pre-image has diameter exactly 3 , then the line graph has no disconnected cut.

Related Work. As mentioned, the name Disconnected Cut stems from Fleischner et al. [15], who studied how to partition the vertices of a graph into exactly $k$ bicliques, where Disconnected Cut is equivalent to the case $k=2$. However, Disconnected Cut originates from $H$-partitions, introduced in [8]. A model graph $H$ on vertices $h_{1}, \ldots, h_{k}$ has solid and dotted edges. An $H$-partition of a graph $G$ is a partition of $V(G)$ into $|V(H)|$ nonempty sets $V_{1}, \ldots, V_{k}$ such that for every pair of vertices $u \in V_{i}$ and $v \in V_{j}$ : if $h_{i} h_{j}$ is a solid edge of $E(H)$, then $u v \in E(G)$; and if $h_{i} h_{j}$ is a dotted edge of $E(H)$, then $u v \notin E(G)$ 
(if $h_{i} h_{j} \notin E(H)$, then $u v \in E(G)$ or $u v \notin E(G)$ are both allowed). The corresponding decision problem is called $H$-PArtition. Dantas et al. [8] proved that $H$-PArtition is polynomial-time solvable for every 4 -vertex model graph $H$ except $H=2 K_{2}$, which has solid edges $h_{1} h_{3}, h_{2} h_{4}$ and no dotted edges, and $H=2 S_{2}$, which has dotted edges $h_{1} h_{3}, h_{2} h_{4}$ and no solid edges. These two cases are polynomial-time equivalent to DisconneCTED Cut. Hence, we now know that, as a matter of exception, $H$-PARTition is NP-complete if $H \in\left\{2 K_{2}, 2 S_{2}\right\}[26]$.

We can encode a model graph $H$ as a matrix $M$ in which every entry is either 0 (dotted edge), 1 (solid edge) or $*$ (no restriction). If we allow sets $V_{i}$ in a solution for $H$-PARTition to be empty, then we obtain the $M$-PARTiTion problem, introduced by Feder et al. [13]. This well-known problem generalizes many classical problems involving vertex cuts and partitions, including $k$-COLOURING and $H$-Colouring; see also [18]. An even more general variant is to give every vertex $u$ a list $L(u) \subseteq\{1, \ldots, k\}$ and to search for a solution, in which each vertex $u$ may only belong to a set $V_{i}$ with $i \in L(u)$. This yields the List $M$-PARTITION problem, which includes well-known cases, such as the STUBBoRn problem, which turned out to be polynomial-time solvable [7], in contrast to Disconnected Cut. A homomorphism $f$ from $G$ to $H$ is a retraction if $G$ contains $H$ as an induced subgraph and $f(u)=u$ for every $u \in V(H)$. The corresponding decision version is called $H$-REtraCtion. Let $\mathcal{C}_{4}$ be the 4-cycle with a self-loop in each vertex. Then $\mathcal{C}_{4}$-RETRACTION is a special case of LIST $2 S_{2}$-PARTITION where the input graph contains a cycle on four specified vertices $v_{1}, \ldots, v_{4}$ with $L\left(v_{i}\right)=\{i\}$ for $i=1, \ldots, 4$ and $L(v)=\{1,2,3,4\}$ for $v \notin\left\{v_{1}, \ldots, v_{4}\right\}$. This problem is a generalization of Disconnected Cut. Feder and Hell [12] proved that $\mathcal{C}_{4}$-Retraction is NP-complete. Hence, List $2 S_{2}$-PARTition and List $2 K_{2}$-PARTition are NP-complete. Note that this result is also implied by the NP-completeness of $2 K_{2}$-PARTition [26].

Vikas [29] solved an open problem of Winkler (see [13, 29]) by proving NP-completeness of $\mathcal{C}_{4}$-Compaction, the variant of the $2 S_{2}$-PARTition problem with the extra constraint that there must be at least one edge $u_{i} u_{j}$ with $u_{i} \in V_{i}$ and $u_{i+1} \in V_{i+1}$ for $i=1, \ldots, 4$ (where $V_{5}=V_{1}$ ). Generally, a homomorphism $f$ from a graph $G$ to a graph $H$ is a compaction if $f$ is edge-surjective, i.e., for every $x y \in E(H)$ with $x \neq y$ there is an edge $u v \in E(G)$ with $f(u)=x$ and $f(v)=y$. The corresponding decision problem is called $H$-Compaction. If $H=\mathcal{C}_{4}$, then the problem is equivalent to DisconneCted Cut when restricted to graphs of diameter 2 [15]. Hence, $\mathcal{C}_{4}$-Compaction is NP-complete for graphs of diameter 2 [26] (the result of [29] holds for graphs of diameter at least 3). Similarly, a homomorphism $f$ from a graph $G$ to a graph $H$ is (vertex-) surjective if for every $x \in V(H)$ there is a vertex $u \in V(G)$ such that $f(u)=x$. The decision problem is called Surjective $H$-Colouring (or $H$-Vertex Compaction, or Surjective $H$-Homomorphism) and is equivalent to Disconnected Cut if $H=\mathcal{C}_{4}$. The complexity classifications of $H$-CompaCtion and SuRJECTIVE $H$-Colouring are wide open despite many partial results; see [1] for a survey and [16] for a more recent overview focussing on SURJECTIVE $H$-COLOURING .

\section{Preliminaries and Basic Results}

In the remainder of our paper, graphs are finite, undirected, and have neither multiple edges nor self-loops unless explicitly stated otherwise. Let $G=(V, E)$ be a graph. For a set $S \subseteq V$, $G[S]$ is the subgraph of $G$ induced by $S$. We say that $S$ is connected if $G[S]$ is connected. We write $G-S=G[V \backslash S]$, and if $S=\{u\}$, we write $G-u$ instead. For a vertex $u \in V$, let $N(u)=\{v \mid u v \in E\}$ be the neighbourhood of $u$ and $N[u]=N(u) \cup\{u\}$. The complement 
$\bar{G}$ of $G$ has vertex set $V$ and edge set $\{u v \mid u v \notin E\}$. The distance $d_{G}(u, v)$ between vertices $u$ and $v$ of $G$ is the number of edges in a shortest path between them. The diameter of $G$ is equal to $\max \left\{d_{G}(u, v) \mid u, v \in V\right\}$. The following lemma was observed by Fleischner et al.

- Lemma 1 ([15]). If a graph $G$ has diameter 1 , then $G$ has no disconnected cut. If a graph $G$ has diameter at least 3 , then $G$ has a disconnected cut, which can be found in linear time.

A subset $D \subseteq V$ is a dominating set of a graph $G=(V, E)$ if every vertex of $V \backslash D$ is adjacent to at least one vertex of $D$. If $D=\{u\}$, then $u$ is a dominating vertex of $G$. A vertex $u \in V$ has a disconnected neighbourhood if $N(u)$ induces a disconnected graph.

- Lemma 2. If a graph $G$ contains a dominating vertex, then $G$ has no disconnected cut.

- Lemma 3 (proof omitted). If a graph $G$ contains a non-dominating vertex $u$ with a disconnected neighbourhood, then $G$ has a disconnected cut.

Two disjoint vertex sets $S$ and $T$ in a graph $G=(V, E)$ are complete to each other if there is an edge between every vertex of $S$ and every vertex of $T$, and $S$ and $T$ are anticomplete to each other if there is no edge between a vertex of $S$ and a vertex of $T$. Recall that $G$ has a disconnected cut if $V$ can be partitioned into four nonempty sets $V_{1}, V_{2}, V_{3}, V_{4}$, such that $V_{1}$ is anticomplete to $V_{3}$ and $V_{2}$ is anticomplete to $V_{4}$. We say that $V_{1}, V_{2}, V_{3}, V_{4}$ form a disconnected partition of $G$.

- Lemma 4. Let $V_{1}, V_{2}, V_{3}, V_{4}$ be a disconnected partition of a graph $G$ of diameter 2 . Then $G$ has an induced cycle $C$ with $4 \leq|V(C)| \leq 5$ such that $V(C) \cap V_{i} \neq \emptyset$ for $i=1, \ldots, 4$.

Proof. Let $u_{1} \in V_{1}$ and $u_{3} \in V_{3}$. As $G$ has diameter 2 , there exists a vertex $u_{2}$ in $V_{2}$ or $V_{4}$, say $V_{2}$, such that $u_{2}$ is adjacent to $u_{1}$ and to $u_{3}$. Let $u_{4} \in V_{4}$. As $G$ has diameter 2 , there exists a vertex $u_{1}^{\prime}$ in $V_{1}$ or $V_{3}$, say $V_{1}$, such that $u_{1}^{\prime}$ is adjacent to $u_{2}$ and $u_{4}$. If $u_{3}$ and $u_{4}$ are adjacent, then we can take as $C$ the cycle on vertices $u_{1}^{\prime}, u_{2}, u_{3}, u_{4}$ in that order. Otherwise, as $G$ has diameter 2, there exists a vertex $w \in V_{3} \cup V_{4}$, such that $w$ is adjacent to $u_{3}$ and to $u_{4}$. In that case we can take as $C$ the cycle on vertices $u_{1}^{\prime}, u_{2}, u_{3}, w, u_{4}$.

Two adjacent vertices $u$ and $v$ of graph $G=(V, E)$ have a nested neighbourhood if $N(u) \backslash\{v\} \subseteq N(v) \backslash\{u\}$ or $N(v) \backslash\{u\} \subseteq N(u) \backslash\{v\}$. We say that $G$ has distinct neighbourhoods if $G$ has no two vertices that have nested neighbourhoods.

- Lemma 5 (proof omitted). Let $G$ be a graph of diameter 2 that contains two vertices $u$ and $v$ such that $N(u) \backslash\{v\} \subseteq N(v) \backslash\{u\}$. Then $G$ has a disconnected cut if and only if $G-u$ has a disconnected cut. Moreover, $G-u$ has diameter at most 2.

A pair of vertices $u$ and $v$ of a graph $G=(V, E)$ is a universal pair if $\{u, v\}$ is a dominating set and there exist distinct vertices $x$ and $y$ in $V \backslash\{u, v\}$, such that $x \in N(u)$ and $y \in N(v)$; note that this implies that $|V| \geq 4$ and $u, v$ have at least one neighbour in $V-\{u, v\}$. Let $H$ be a graph. Then $G$ is $H$-free if $G$ contains no induced subgraph isomorphic to $H$. The disjoint union $G+H$ of two vertex-disjoint graphs $G$ and $H$ is the graph $(V(G) \cup V(H), E(G) \cup E(H))$. The disjoint union of $r$ copies of a graph $G$ is denoted by $r G$. The graphs $C_{r}$ and $P_{r}$ denote the cycle and path on $r$ vertices, respectively. The graph $K_{r}$ denotes the complete graph on $r$ vertices. The independence number $\alpha(G)$ of a graph $G$ is the largest $k$ such that $G$ contains an induced subgraph isomorphic to $k P_{1}$.

- Lemma 6 ([6]). A $2 P_{2}$-free graph has a disconnected cut if and only if its complement has a universal pair. 
- Lemma 7 ([9]). Disconnected Cut is $O\left(n^{3}\right)$-time solvable for $4 P_{1}$-free graphs.

The graph $\left(\left\{u, v_{1}, v_{2}, v_{2}\right\},\left\{u v_{1}, u v_{2}, u v_{3}\right\}\right)$ is the claw $K_{1,3}$. A graph is cobipartite if it is the complement of a bipartite graph. The line graph of a graph $G$ with edges $e_{1}, \ldots, e_{p}$ is the graph $L(G)$ with vertices $u_{1}, \ldots, u_{p}$ such that there is an edge between any two vertices $u_{i}$ and $u_{j}$ if and only if $e_{i}$ and $e_{j}$ have a common endpoint in $G$. Note that every line graph is claw-free. We call $G$ the preimage of $L(G)$. Every connected line graph except $K_{3}$ has a unique preimage [17]. A circular-arc graph is a graph that has a representation in which each vertex corresponds to an arc of a circle, such that two vertices are adjacent if and only if their corresponding arcs intersect. An interval graph is a graph that has representation in which each vertex corresponds to an interval of the line, such that two vertices are adjacent if and only if their corresponding intervals intersect. Note that circular-arc graphs generalize interval graphs. A circular-arc or interval graph is proper if it has a representation where the arcs respectively intervals are such that no one is contained in another.

\section{Circular-Arc Graphs}

In this section we prove that DisConNeCTED CUT is polynomial-time solvable for circular-arc graphs. This result is known already for interval graphs, as it follows from the result that Disconnected Cut is polynomial-time solvable for the class of chordal graphs [22], which contains the class of interval graphs. In fact, we have an $O\left(n^{2}\right)$-time algorithm for interval graphs. Due to Lemma 4 and the fact that interval graphs are chordal, no interval graph of diameter 2 has a disconnected cut. Consequently, an interval graph has a disconnected cut if and only if its diameter is at least 3 due to Lemma 1. To show that Disconnected CuT is polynomial-time solvable for circular-arc graphs requires significant additional work.

Let $G$ be a circular-arc graph. For each vertex $u \in V(G)$ we can associate an arc $\left[l_{u}, r_{u}\right]$ where we say that $l_{u}$ is the clockwise left endpoint of $u$ and $r_{u}$ is the clockwise right endpoint of $u$. We may assume that all left and right endpoints of the vertices of $G$ are unique.

- Lemma 8 ([27]). A circular-arc graph $G$ on $n$ vertices and $m$ edges can be recognized in $O(n+m)$ time. In the same time, a representation of $G$ can be constructed with distinct arc endpoints that are clockwise enumerated as $1, \ldots, 2 n$.

For the main result in the section we need the following lemma (proof omitted).

- Lemma 9. Let $G$ be a circular-arc graph of diameter 2 with a disconnected cut. Then $G$ has a disconnected partition $V_{1}, V_{2}, V_{3}, V_{4}$ such that each $V_{i}$ is connected.

- Theorem 10. Disconnected Cut is $O\left(n^{2}\right)$-time solvable for circular-arc graphs.

Proof Sketch. Let $G=(V, E)$ be a circular-arc graph on $n$ vertices. We will either find a disconnected cut or conclude that $G$ has no disconnected cut. We compute the diameter of $G$ in $O\left(n^{2}\right)$ time, say by using the (more general) $O\left(n^{2}\right)$-time algorithm of [4]. By Lemma 1, we may assume that $G$ has diameter 2. Lemma 9 tells us that if $G$ has a disconnected cut, then $G$ has a disconnected partition $V_{1}, V_{2}, V_{3}, V_{4}$ such that $V_{i}$ is connected for $i=1, \ldots, 4$. We say that the arc of a set $V_{i}$ is the union of all the arcs of the vertices in $V_{i}$. As $G$ has diameter 2, the union of the arcs of the sets $V_{i}$ covers the whole circle. Moreover, the arcs of $V_{1}$ and $V_{3}$ are disjoint and the arcs of $V_{2}$ and $V_{4}$ are disjoint.

We now compute, in linear time, a representation of $G$ with distinct arc endpoints clockwise enumerated as $1, \ldots, 2 n$ via Lemma 8. We then sort the $\operatorname{arcs}$ in $O(n \log n)$ time. Then, in $O\left(n^{2}\right)$ time, we check if there is a pair or triple of vertices whose arcs cover the whole circle. If so, then using Lemma 9 we find that $G$ has no disconnected cut. 
Suppose $G$ has no pair or triple of vertices whose arcs cover the whole circle. Then, in $O(n)$ time, we find an induced cycle $v_{1}, \ldots, v_{k}$ whose arcs cover the whole circle and such that $k \in\{4,5\}$. If $G$ has a disconnected partition $V_{1}, V_{2}, V_{3}, V_{4}$ such that each $V_{i}$ is connected, then we have the following. If $k=4$, then we may assume without loss of generality that $v_{i} \in V_{i}$ for $i=1, \ldots, 4$. If $k=5$, then two vertices $v_{i}, v_{i+1}$ belong to the same set $V_{h}$, whereas sets $V_{i}$ with $i \neq h$ each contain a single vertex from $C$. If $k=5$, then we guess which two vertices $v_{i}, v_{i+1}$ will be put in the same set, say $v_{1}, v_{5}$; this does not influence the asymptotic running time.

Now we build up the sets $V_{i}$ from scratch by putting in the vertices from $V(G) \backslash\left\{v_{1}, \ldots, v_{k}\right\}$. We maintain that each $V_{i}$ induces a connected graph, and thus, the union of the arcs of the vertices in $V_{i}$ indeed always form an arc. Observe also that no set $V_{h}$ is contained in some other set $V_{i}$; by our choice of vertices $v_{i}$ we will always maintain this property. We say that a vertex $u$ intersects a set $V_{i}$ if the arc of $u$ intersects the arc of $V_{i}$. Note that, since the arcs corresponding to $\left\{v_{1}, \ldots, v_{k}\right\}$ cover the entire circle, so do the arcs of the sets $V_{i}$ that we are constructing. If there is a vertex that intersects each of the sets $V_{i}$ constructed so far, then there is no disconnected cut with each $V_{i}$ connected. If $k=4$, then $G$ has no disconnected cut due to Lemma 9 . If $k=5$, then our guess of vertices $v_{1}, v_{5}$ to belong to $V_{h}$ may have been incorrect, and we need to put two other consecutive vertices of $C$ in the same set $V_{h}$ before concluding that $G$ has no disconnected cut. Otherwise, we do the following until no longer possible. As no $V_{h}$ is contained in some other $V_{h}$, any vertex $u$ that intersects two sets $V_{i}$ and $V_{i+2}$ for some $i$ (say, $i \in\{1,2\}$ without loss of generality), also intersects $V_{i+1}$ or $V_{i+3}$ (where $V_{5}=V_{1}$ ). We put a vertex $u$ that intersects two sets $V_{i}$ and $V_{i+2}$ for some $i$ into set $V_{i+1}$ if $u$ intersects $V_{i+1}$ as well; otherwise, $u$ intersects $V_{i+3}$ and we put $u$ in $V_{i+3}$.

Let $T$ be the set of vertices of $G$ that we have not placed in some set $V_{i}$ yet. We show that each vertex of $T$ must intersect exactly two sets $V_{i}$ and $V_{j}$ such that, in addition, $j=i+1$ holds. Then we can model the remaining instance as an instance of 2-SATISFIABILITY and solve it in $O\left(n^{2}\right)$ time.

\section{$4 \quad$ Line Graphs}

In this section we prove that DisConneCTED CUT is polynomial-time solvable for line graphs. We start with the following lemma due to Ito et al. [22].

- Lemma 11 ([22]). Let $G$ be a graph with diameter 2 whose line graph $L(G)$ also has diameter 2. Then $G$ has a disconnected cut if and only if $L(G)$ has a disconnected cut.

For the main result in the section we need the following lemma (proof omitted).

- Lemma 12. Let $G$ be a graph that is neither a triangle nor a star. Then $L(G)$ has diameter 2 if and only if $G$ is $2 P_{2}$-free.

Theorem 13. DisCONnECTED Cut is $O\left(n^{4}\right)$-time solvable on line graphs of n-vertex graphs.

Proof. Let $G$ be a graph on $n$ vertices and $m$ edges. We first check in $O(n)$ time if $G$ is a triangle or star. If so, then $L(G)$ is a complete graph and thus $L(G)$ has no disconnected cut. From now on suppose that $G$ is neither a triangle nor a star. By Lemma 12 we find that $L(G)$ has diameter 2 if and only if $G$ is $2 P_{2}$-free. Hence, we can check in $O\left(n^{4}\right)$ time, via checking if $G$ has an induced $2 P_{2}$ by brute force, if $L(G)$ has diameter 2 .

First assume that $L(G)$ does not have diameter 2. As $G$ is not a triangle or a star, $L(G)$ has diameter at least 3. By Lemma 1 we find that $L(G)$ has a disconnected cut. Now assume that $L(G)$ has diameter 2. We check in $O\left(n^{3}\right)$ time if $G$ has an edge $u v$ such that every 
vertex of $V(G) \backslash\{u, v\}$ is adjacent to at least one of $u, v$. If so, then $u v$ is a dominating vertex of $L(G)$, and $L(G)$ has no disconnected cut due to Lemma 2. If not, then $L(G)$ has no dominating vertices, and we proceed as follows. First we check if $L(G)$ has a vertex $u v$ with a disconnected neighbourhood, or equivalently, if $G$ contains an edge $u v$ such that $u$ and $v$ have degree at least 2 and no common neighbours. This takes $O\left(n^{3}\right)$ time. If $L(G)$ has a vertex with a disconnected neighbourhood, then $L(G)$ has a disconnected cut by Lemma 3 . From now on assume that $L(G)$ has no vertex with a disconnected neighbourhood. As $G$ is neither a triangle nor a star, $G$ is $2 P_{2}$-free by Lemma 12 . Hence, $G$ has diameter at most 3 . We can determine in $O\left(n^{3}\right)$ time the diameter of $G$ and consider each case separately.

Case 1. $G$ has diameter 1 .

We claim that $L(G)$ has no disconnected cut. For contradiction, assume that $L(G)$ has a disconnected cut. Let $V_{1}^{\prime}, V_{2}^{\prime}, V_{3}^{\prime}, V_{4}^{\prime}$ be a disconnected partition of $L(G)$. By Lemma $4, L(G)$ contains a cycle $C^{\prime}$ with vertices $u_{i} u_{i+1}$ for $i=1, \ldots, j$ (with $u_{j+1}=u_{1}$ ) and $j \in\{4,5\}$, such that $V\left(C^{\prime}\right) \cap V_{i}^{\prime} \neq \emptyset$ for $i=1,2,3,4$. Then we may assume without loss of generality that $u_{i} u_{i+1} \in V_{i}^{\prime}$ for $i=1, \ldots, 4$ and $u_{j} u_{j+1} \in V_{4}^{\prime}$. As $G$ has diameter $1, u_{1} u_{3}$ is an edge of $G$ and thus a vertex of $L(G)$. In $L(G), u_{1} u_{3}$ is adjacent to every vertex in $\left\{u_{1} u_{2}, u_{2} u_{3}, u_{3} u_{4}, u_{j} u_{j+1}\right\}$, and thus to a vertex in $V_{i}^{\prime}$ for $i=1, \ldots, 4$, a contradiction.

Case 2. $G$ has diameter 2 .

Then $G$ has a disconnected cut if and only if $L(G)$ has a disconnected cut due to Lemma 11 . By Lemma 6 it suffices to check if $\bar{G}$ has a universal pair. This takes $O\left(n^{3}\right)$ time.

Case 3. $G$ has diameter 3 .

We will prove that $L(G)$ has no disconnected cut. As $G$ has diameter $3, G$ does have a disconnected cut by Lemma 1 . We need the following claim.

- Claim. Let $V_{1}, V_{2}, V_{3}, V_{4}$ be a disconnected partition of $G$. Then every cycle $C$ of $G$ with $4 \leq|V(C)| \leq 5$ contains vertices of at most three distinct sets from $\left\{V_{1}, V_{2}, V_{3}, V_{4}\right\}$.

We prove the Claim as follows. For contradiction, assume that $G$ has a cycle $C$ with vertices $u_{1}, \ldots, u_{j}$ for $j \in\{4,5\}$, such that $V(C) \cap V_{i} \neq \emptyset$ for $i=1, \ldots, 4$. We may assume without loss of generality that $u_{i} \in V_{i}$ for $i=1, \ldots, 4$ and $u_{j} \in V_{4}$. As $G$ is $2 P_{2}$-free, we may assume without loss of generality that $u_{3}$ is in a singleton connected component of $G\left[V_{3}\right]$. If $j=4$, then we may also assume without loss of generality that $u_{2}$ is in a singleton connected component of $G\left[V_{2}\right]$. If $j=5$, then $u_{2}$ must be in a singleton connected component of $G\left[V_{2}\right]$ due to the edge $u_{4} u_{5}$, which is contained in $G\left[V_{4}\right]$. This means that the sets $N_{G}\left(u_{2}\right) \backslash\left\{u_{3}\right\}$ and $N_{G}\left(u_{3}\right) \backslash\left\{u_{2}\right\}$ are disjoint. As $u_{1} u_{2}$ and $u_{3} u_{4}$ are edges of $G$, both $\left.N_{G}\left(u_{2}\right) \backslash\left\{u_{3}\right\}\right\}$ and $\left.N_{G}\left(u_{3}\right) \backslash\left\{u_{2}\right\}\right\}$ are nonempty. Hence, the vertex $u_{2} u_{3}$ has a disconnected neighbourhood in $L(G)$, a contradiction. This proves the Claim.

Now, for contradiction, assume that $L(G)$ has a disconnected cut. Let $V_{1}^{\prime}, V_{2}^{\prime}, V_{3}^{\prime}, V_{4}^{\prime}$ be a disconnected partition of $L(G)$. By Lemma $4, L(G)$ contains a cycle $C^{\prime}$ with vertices $u_{i} u_{i+1}$ for $i=1, \ldots, j$ (with $u_{j+1}=u_{1}$ ) and $j \in\{4,5\}$, such that $V\left(C^{\prime}\right) \cap V_{i}^{\prime} \neq \emptyset$ for $i=1,2,3,4$. Assume without loss of generality that $u_{i} u_{i+1} \in V_{i}^{\prime}$ for $i=1, \ldots, 4$ and $u_{j} u_{j+1} \in V_{4}^{\prime}$.

We define the following partition $V_{1}, V_{2}, V_{3}, V_{4}$ of $V(G)$. Let $u \in V(G)$. If $u$ is incident to only edges from one set $V_{i}^{\prime}$, then we put $u$ in $V_{i}$. Suppose $u$ is incident to edges from more than one set $V_{i}^{\prime}$. As $V_{1}^{\prime}, V_{2}^{\prime}, V_{3}^{\prime}, V_{4}^{\prime}$ is a disconnected partition of $L(G)$, we find that $u$ is incident to edges from $V_{i}^{\prime}$ and $V_{i+1}^{\prime}$ for some $1 \leq i \leq 4$ (where $V_{5}=V_{1}$ ) and to no other sets $V_{j}^{\prime}$. In that case we put $u$ into $V_{i+1}$. 
We now prove that $V_{1}$ is anticomplete to $V_{3}$. For contradiction, assume that $V_{1}$ contains a vertex $u$ and $V_{3}$ contains a vertex $v$ such that $u v \in E(G)$. As $u \in V_{1}$, we find that $u$ is incident to edges only in $V_{4}^{\prime}$ and $V_{1}^{\prime}$. Hence, $u v \in V_{1}^{\prime} \cup V_{4}^{\prime}$. As $v \in V_{3}$, we find that $v$ is incident to edges only in $V_{2}^{\prime}$ and $V_{3}^{\prime}$. This implies that $u v \in V_{2}^{\prime} \cup V_{3}^{\prime}$, a contradiction. By the same argument we can show that $V_{2}$ is anticomplete to $V_{4}$. Let $C$ be the cycle with vertices $u_{1}, \ldots, u_{j}$ in $G$. Then $V(C) \cap V_{i} \neq \emptyset$, and thus $V_{i} \neq \emptyset$, for $i=1, \ldots, 4$. Hence, $V_{1}, V_{2}, V_{3}$, $V_{4}$ is a disconnected partition of $G$, and $C$ is a cycle in $G$ with $V(C) \cap V_{i} \neq \emptyset$ for every $i$. This is not possible due to the Claim. We conclude that $L(G)$ has no disconnected cut.

The correctness of our algorithm follows from the above. If $G$ has diameter 1 (Case 1) or diameter 3 (Case 3), no additional running time is required, as $L(G)$ has no disconnected cut in both these cases. Hence, only executing Case 2 takes additional time, namely time $O\left(n^{3}\right)$. Hence, the total running time of our algorithm is $O\left(n^{4}\right)$.

\section{$5 \quad$ Claw-Free Graphs}

In this section, we prove that Disconnected CUT is polynomial-time solvable on claw-free graphs. The proof consists of two parts. In Section 5.1 we show how to get rid of certain cobipartite structures in the graph, called W-joins. We remark that Disconnected CuT can be solved in polynomial time on cobipartite graphs [15]. Although this is a necessary condition for DisconneCted Cut to be solvable in polynomial time on claw-free graphs, the algorithm for cobipartite graphs is not sufficient to deal with $\mathrm{W}$-joins. In Section 5.2 we present our new decomposition theorem for claw-free graphs of diameter 2 and combine this theorem with the results from the previous sections and Section 5.1 to show our main result.

\subsection{Cobipartite Structures versus Disconnected Cuts}

A pair $(A, B)$ of disjoint non-empty sets of vertices is a $W$-join in graph $G$ if $|A|+|B|>2$, $A$ and $B$ are cliques, $A$ is neither complete nor anticomplete to $B$, and every vertex of $V(G) \backslash(A \cup B)$ is either complete or anticomplete to $A$ and either complete or anticomplete to $B$. A W-join is a proper $W$-join if each vertex in $A$ is neither complete nor anticomplete to $B$ and each vertex in $B$ is neither complete nor anticomplete to $A$. Observe that for a proper $\mathrm{W}$-join $(A, B)$, it must hold that $|A|,|B| \geq 2$. For any $\mathrm{W}$-join $(A, B)$, it holds that $G[A \cup B]$ is a cobipartite induced subgraph in $G$.

We assume that an input graph $G$ of Disconnected Cut has diameter 2 and that $G$ has distinct neighbourhoods, by Lemmas 1 and 5 respectively. We show how to use these assumptions to remove all $\mathrm{W}$-joins in a claw-free graph and obtain an equivalent instance of Disconnected Cut. As a first step, we show that we can focus on proper W-joins.

- Lemma 14 (proof omitted). Let $G$ be a graph with distinct neighbourhoods. If $G$ admits a $W$-join $(A, B)$, then $(A, B)$ is a proper $W$-join.

A W-join $(A, B)$ is partitionable if there are partitions of $A$ into non-empty sets $A^{\prime}, A^{\prime \prime}$ and of $B$ into non-empty sets $B^{\prime}, B^{\prime \prime}$ such that $A^{\prime}$ is anticomplete to $B^{\prime \prime}$ and $B^{\prime}$ is anticomplete to $A^{\prime \prime}$. A proper $\mathrm{W}$-join $(A, B)$ is shatterable if it is partitionable with sets $A^{\prime}, A^{\prime \prime}, B^{\prime}, B^{\prime \prime}$ and one of $\left(A^{\prime}, B^{\prime}\right),\left(A^{\prime \prime}, B^{\prime \prime}\right)$ is also a proper $\mathrm{W}$-join; we say it is unshatterable otherwise.

- Lemma 15 (proof omitted). Let $G$ be a graph with distinct neighbourhoods and let $(A, B)$ be a proper $W$-join in $G$. If $(A, B)$ is partitionable and unshatterable, then $G[A \cup B]$ is isomorphic to $C_{4}$. 
- Lemma 16 (proof omitted). Let $G$ be a claw-free graph that is not cobipartite, has distinct neighbourhoods, and has diameter 2 . Let $(A, B)$ be a proper $W$-join in $G$ that is unshatterable. If $G$ admits a disconnected cut, then there exists a disconnected partition $V_{1}, V_{2}, V_{3}, V_{4}$ of $G$ such that $V_{i} \cap(A \cup B)=\emptyset$ for some $i \in\{1,2,3,4\}$.

Let $(A, B)$ be a proper W-join of a graph $G$. For any two adjacent vertices $a \in A$ and $b \in B$, let $G_{a b}$ be the graph obtained from $G$ by removing $A \backslash\{a\}$ and $B \backslash\{b\}$. Observe that the graph $G_{a b}$ is the same regardless of the choice of $a, b$.

- Lemma 17. Let $G$ be a claw-free graph that is not cobipartite, has distinct neighbourhoods, and has diameter 2 . Let $(A, B)$ be a proper $W$-join of $G$ that is unshatterable. Then $G$ admits a disconnected cut if and only if $G_{a b}$ admits a disconnected cut for any two adjacent vertices $a \in A$ and $b \in B$.

Proof. First suppose that $G_{a b}$ admits a disconnected partition $V_{1}, V_{2}, V_{3}, V_{4}$ for any two vertices $a, b$. Let $a \in V_{i}$ and $b \in V_{j}$ for $i, j \in\{1,2,3,4\}$. Then the sets $V_{1}^{\prime}, V_{2}^{\prime}, V_{3}^{\prime}, V_{4}^{\prime}$ obtained from $V_{1}, V_{2}, V_{3}, V_{4}$ by adding $A$ to $V_{i}$ and $B$ to $V_{j}$ is a disconnected partition of $G$.

Now suppose that $G$ admits a disconnected cut. Let $V_{1}, V_{2}, V_{3}, V_{4}$ be a disconnected partition of $G$. By Lemma 16, we may assume without loss of generality that $V_{4} \cap(A \cup B)=\emptyset$. Note that $A$ is a clique in $G$ and $V_{1}$ is anticomplete to $V_{3}$, and thus $A \subseteq V_{1} \cup V_{2}$ or $A \subseteq V_{2} \cup V_{3}$. We assume the former without loss of generality. Among all such disconnected partitions, we will assume that $V_{1}, V_{2}, V_{3}, V_{4}$ was chosen to minimize $\left|A \cap V_{1}\right|$.

We consider several cases. In each of these cases we find two vertices $a, b$ for which we can construct a disconnected partition of $G_{a b}$. Note that this suffices to prove the statement, as the graph $G_{a b}$ is the same regardless of the choice of $a, b$.

First assume that $A \subseteq V_{1}$. Since no vertex of $B$ is anticomplete to $A$ by the definition of a proper $\mathrm{W}$-join and $V_{1}$ is anticomplete to $V_{3}$, it follows that $B \subseteq V_{1} \cup V_{2}$. Now if $B \subseteq V_{1}$, then let $a \in A$ and $b \in B$ be arbitrary adjacent vertices (these exist by the definition of a W-join) and $V_{1} \backslash((A \backslash\{a\}) \cup(B \backslash\{b\})), V_{2}, V_{3}, V_{4}$ is a disconnected partition of $G_{a b}$. Otherwise, let $b \in B \cap V_{2}$ and let $a$ be an arbitrary vertex of $A$ that is adjacent to $b$ (which exists by the definition of a proper W-join). Then $V_{1} \backslash((A \backslash\{a\}) \cup B), V_{2} \backslash(B \backslash\{b\}), V_{3}, V_{4}$ is a disconnected partition of $G_{a b}$.

Now assume that $A \subseteq V_{2}$. Note that $B \subseteq V_{1} \cup V_{2} \cup V_{3}$. Since $B$ is a clique and $V_{1}$ is anticomplete to $V_{3}$, it follows that $B \subseteq V_{1} \cup V_{2}$ or $B \subseteq V_{2} \cup V_{3}$. First, assume that $B \subseteq V_{2}$. Let $a \in A$ and $b \in B$ be arbitrary adjacent vertices; note that $a, b \in V_{2}$. Then $V_{1}, V_{2} \backslash((A \backslash\{a\}) \cup(B \backslash\{b\})), V_{3}, V_{4}$ is a disconnected partition of $G_{a b}$. So we may assume that $B \nsubseteq V_{2}$. Then $B \cap V_{1} \neq \emptyset$ or $B \cap V_{3} \neq \emptyset$. Without loss of generality, we assume it is the former. Let $b \in B \cap V_{1}$ and let $a \in A$ be any neighbour of $b$. Note that $a \in V_{2}$. Then $V_{1} \backslash(B \backslash\{b\}), V_{2} \backslash((A \backslash\{a\}) \cup B), V_{3}, V_{4}$ is a disconnected partition of $G_{a b}$.

It remains to consider the case where $A \cap V_{1} \neq \emptyset$ and $A \cap V_{2} \neq \emptyset$. Let $P=N(A) \backslash N[B]$, $Q=N(B) \backslash N[A], M=N[A \cup B] \backslash(P \cup Q)$, and $R=V(G) \backslash(P \cup Q \cup M)$. Note that $P$ is complete to $A$ and anticomplete to $B$, whereas $Q$ is complete to $B$ and anticomplete to $A$. Moreover, $M$ is complete to $A \cup B$, whereas $R$ is anticomplete to $A \cup B$. Then, by the assumptions of the case, we have that $P \subseteq V_{1} \cup V_{2}$. Note that $B \subseteq V_{1} \cup V_{2} \cup V_{3}$. Since $B$ is a clique and $V_{1}$ is anticomplete to $V_{3}$, it follows that $B \subseteq V_{1} \cup V_{2}$ or $B \subseteq V_{2} \cup V_{3}$. Moreover, as $A \cap V_{1} \neq \emptyset$, it follows from the definition of a proper $\mathrm{W}$-join that $B \nsubseteq V_{3}$. We now prove that $B \subseteq V_{1} \cup V_{2}$.

For contradiction, assume that $B \cap V_{3} \neq \emptyset$ and thus $B \cap V_{2} \neq \emptyset$. As $M$ is complete to $A$ and $B$ and $A \cup B$ has a nonempty intersection with each of $V_{1}, V_{2}, V_{3}$, it follows from the definition of a disconnected partition that $M \subseteq V_{2}$. Similarly, we derive that 
$Q \subseteq V_{2} \cup V_{3}$; recall also that $P \subseteq V_{1} \cup V_{2}$. Suppose $V_{1} \backslash A \neq \emptyset$. Then $V_{1} \backslash A, V_{2} \cup A, V_{3}, V_{4}$ is also a disconnected partition of $G$, contradicting our choice of the disconnected partition $V_{1}, V_{2}, V_{3}, V_{4}$. Hence, $V_{1} \backslash A=\emptyset$ and thus, $V_{1} \subseteq A$. Then $P \subseteq V_{2}$. By the definition of a W-join, any path of length 2 from a vertex in $R$ to a vertex in $A$ must intersect $P$ or $M$. As $M \cup P \subseteq V_{2}$ and $V_{4}$ is anticomplete to $V_{2}$, we obtain $R \cap V_{4}=\emptyset$. Since $A \cup B \cup P \cup M \cup Q \cup R=V(G)$ and none of $A, B, P, M, Q, R$ intersects $V_{4}$, it follows that $V_{4}=\emptyset$, a contradiction.

We may thus assume that $B \subseteq V_{1} \cup V_{2}$. First, assume that there exist adjacent vertices $a \in A$ and $b \in B$ such that $\left|V_{1} \cap\{a, b\}\right|=1$ (and thus $\left.\left|V_{2} \cap\{a, b\}\right|=1\right)$. Then $V_{1} \backslash((A \cup$ $B) \backslash\{a, b\}), V_{2} \backslash((A \cup B) \backslash\{a, b\})$ is a disconnected partition of $G_{a b}$. Hence, we may assume that no such two vertices exist. It follows that neither $B \subseteq V_{1}$ nor $B \subseteq V_{2}$; otherwise, such $a$ and $b$ would exist by the definition of a proper $\mathrm{W}$-join. Then we may conclude that $(A, B)$ is partitionable with sets $A \cap V_{1}, A \cap V_{2}, B \cap V_{1}, B \cap V_{2}$. Since $(A, B)$ is unshatterable, it follows from Lemma 15 that $G[A \cup B]$ is isomorphic to $C_{4}$. Hence, $|A|=|B|=2$ and $V_{1}, V_{2}$ each contain exactly one vertex of $A$ and exactly one vertex of $B$. Since $G$ is not cobipartite and $G$ is connected (as $G$ has diameter 2), it follows that one of $P, M, Q$ is non-empty. However, each vertex in $P \cup M \cup Q$ is adjacent to a vertex of $V_{1}$ and a vertex of $V_{2}$. Hence, $P \cup M \cup Q \subseteq V_{1} \cup V_{2}$. Without loss of generality, $(P \cup M \cup Q) \cap V_{1} \neq \emptyset$. Let $a$ be the single vertex of $A \cap V_{2}$ and let $b$ be the single vertex of $B \cap V_{2}$. Then $V_{1} \backslash(A \cup B), V_{2}, V_{3}, V_{4}$ is a disconnected partition of $G_{a b}$. The lemma follows.

In Section 5.2 we will show that by iterating the above lemma, we can remove all Wjoins from an input claw-free graph of diameter 2. However, to this end, it is crucial to have a polynomial-time algorithm that actually finds an unshatterable proper $\mathrm{W}$-join (if it exists). Our algorithm for this problem relies on the $O\left(n^{2} m\right)$-time algorithm by King and Reed [25] to find a proper $\mathrm{W}$-join $(A, B)$. We test in linear time whether the proper $\mathrm{W}$-join is partitionable by considering the graph $H$ obtained from $G[A \cup B]$ by removing all edges with both endpoints in $A$ or in $B$. We argue that we can recurse on a smaller proper $\mathrm{W}$-join if $H$ has two or more connected components, and that $(A, B)$ is unshatterable otherwise.

- Lemma 18 (proof omitted). Let $G$ be a graph with distinct neighbourhoods. Then in $O\left(n^{2} m\right)$ time, we can find an unshatterable proper $W$-join in $G$, or report that $G$ has no proper $W$-join.

\subsection{Structure of Claw-Free Graphs and Solving Disconnected Cut}

Before our main result we first show a decomposition of claw-free graphs of diameter 2 .

- Theorem 19. Every claw-free graph $G$ of diameter 2 with distinct neighbourhoods, no $W$-joins, $\alpha(G)>3$, and $|V(G)|>13$ is a proper circular-arc graph or a line graph.

Proof Sketch. One of the algorithmic structure theorems for claw-free graphs by Hermelin et al. [19, Theorem 6.8] (see also [20]) essentially shows that a claw-free graph that satisfies the assumptions of the theorem is almost a line graph, but certain vertices of this line graph are replaced with large structures called stripes. A stripe is basically an induced subgraph of the graph with one or two specially marked 'ends'. These ends are cliques, contain the only vertices that are incident with edges that connect the stripe with the rest of the graph, and for each end its neighbourhood outside the stripe is a clique. Using the fact that the diameter is 2 , we argue that if a stripe contains a vertex $x$ that is not in an end of the stripe, then every vertex of $G$ must be in the stripe or in the neighbourhood of its ends. This enables us to prove the main claim in the theorem, which is that the stripe is essentially the whole 
graph if it has such a vertex $x$. After proving the claim, it suffices to consider the different cases in the structural theorem and prove that, by using the claim and the assumptions of the theorem, $G$ must be a proper circular-arc graph or a line graph.

- Theorem 20. Disconnected Cut is $O\left(n^{3} m\right)$-time solvable for claw-free graphs.

Proof. Let $G$ be a connected claw-free graph on $n$ vertices and $m$ edges. We will either find a disconnected cut or conclude that $G$ has no disconnected cut. Assume $n \geq 14$. We compute the diameter of $G$ in $O\left(n^{2}\right)$ time. By Lemma 1, G has no disconnected cut if its diameter is 1 and has a disconnected cut if its diameter is at least 3 . Assume the diameter of $G$ is 2 . We check if $\alpha(G) \leq 3$ in $O(n(m+n \log n))$ time [11]. If so, then we decide if $G$ has a disconnected cut in $O\left(n^{3}\right)$ time by Lemma 7. Assume $\alpha(G)>3$. Hence, $G$ is not cobipartite.

Next, we check whether $G$ contains a vertex $u$ for which there exists a vertex $v$ such that $N(u) \backslash\{v\} \subseteq N(v) \backslash\{u\}$. This takes $O\left(n^{3}\right)$ time. If so, then we remove $u$ from $G$ (and restart the algorithm with the resulting graph, which is still connected and claw-free). This is correct by Lemma 5 . Hence, we may assume that $G$ has distinct neighbourhoods.

Then, we get rid of all W-joins in $G$. Since $G$ has distinct neighbourhoods, it follows from Lemma 14 that every $\mathrm{W}$-join in $G$ is a proper W-join. Using Lemma 18, in $O\left(n^{2} m\right)$ time, we can find an unshatterable W-join in $G$ or correctly decide that $G$ does not admit a proper $\mathrm{W}$-join (and thus no W-join). In the former case, we apply Lemma 17 to the unshatterable proper $\mathrm{W}$-join $(A, B)$ that is found. This takes linear time. We then restart the algorithm on the graph $G_{a b}$ found by Lemma 17 (note that $G_{a b}$ is still connected and claw-free). Since $|A|+|B| \geq 3,\left|V\left(G_{a b}\right)\right|<|V(G)|$ and thus we can recurse at most $n$ times. Hence, we may assume that $G$ admits no W-joins.

Next, we check if $G$ is a circular-arc graph in linear time by Lemma 8 . If so, then we apply Theorem 10 to decide if $G$ has a disconnected cut in $O\left(n^{2}\right)$ time. Hence, we may assume that $G$ is not (proper) circular-arc. By Theorem 19 this means that $G$ is a line graph. Hence, we apply Theorem 13 to decide whether $G$ admits a disconnected cut in $O\left(n^{4}\right)$ time. This finishes the description of the algorithm. The running time is clearly $O\left(n^{3} m\right)$.

Recall from $[15,22]$ that $C_{4}$-Contractibility and $\mathcal{C}_{4}$-Compaction are equivalent to DisconneCted CUT on graphs of diameter 2. We combine these claims with Theorem 19.

- Corollary 21. $C_{4}$-Contractibility and $\mathcal{C}_{4}$-Compaction are $O\left(n^{3} m\right)$-time solvable for claw-free graphs of diameter 2 .

\section{Open Problems}

In light of Corollary 21 we ask about the complexities of $C_{4}$-ContraCtibiLity and $\mathcal{C}_{4^{-}}$ COMPACTION for claw-free graphs of diameter at least 3 . We note that the NP-complete problem $P_{4}$-ContractibiLity [3] is polynomial-time solvable for claw-free graphs [14].

It is not known if there exists a graph $H$ for which $H$-Compaction and SurJective $H$-COLOURING have a different complexity. If we impose restrictions on the input graph, such a graph $H$ is known: $\mathcal{C}_{4}$-Compaction is NP-complete for graphs of diameter 3 [29], whereas SurJective $\mathcal{C}_{4}$-Colouring (being equivalent to Disconnected Cut) is trivial on this graph class. In contrast to claw-free graphs, graphs of diameter 3 do not form a hereditary graph class, that is, they are not closed under vertex deletion. This leads to the natural question if there exist a hereditary graph class $\mathcal{G}$ and a graph $H$, such that $H$-COMPACTION and SurJective $H$-Colouring have different complexity when restricted to $\mathcal{G}$. Should 
$\mathcal{C}_{4}$-Compaction turn out to be NP-complete for claw-free graphs, then due Theorem 19 and the equivalency between Disconnected Cut and Surjective $H$-Colouring we can take the class of claw-free graphs as $\mathcal{G}$ and the graph $\mathcal{C}_{4}$ as $H$ to find such a pair $(\mathcal{G}, H)$.

We also ask what the complexity of DisconneCted CUT is for $K_{4}$-free graphs; as shown in the full version of our paper, the $K_{4}$ is the only 4 -vertex graph $H$ for which this is still open.

\section{References}

1 Manuel Bodirsky, Jan Kára, and Barnaby Martin. The complexity of surjective homomorphism problems - a survey. Discrete Applied Mathematics, 160(12):1680-1690, 2012.

2 Flavia Bonomo, Gianpaolo Oriolo, and Claudia Snels. Minimum weighted clique cover on strip-composed perfect graphs. In Proc. WG 2012, volume 7551 of Lecture Notes in Computer Science, pages 22-33, 2012.

3 Andries E. Brouwer and Henk Jan Veldman. Contractibility and NP-completeness. Journal of Graph Theory, 11(1):71-79, 1987.

4 Danny Z. Chen, D. T. Lee, R. Sridhar, and Chandra N. Sekharan. Solving the all-pair shortest path query problem on interval and circular-arc graphs. Networks, 31(4):249-258, 1998.

5 Maria Chudnovsky and Paul D. Seymour. The structure of claw-free graphs, volume 327 of London Mathematical Society Lecture Note Series, pages 153-171. Cambridge University Press, 2005.

6 Kathryn Cook, Simone Dantas, Elaine M. Eschen, Luérbio Faria, Celina M. H. de Figueiredo, and Sulamita Klein. $2 K_{2}$ vertex-set partition into nonempty parts. Discrete Mathematics, 310(6-7):1259-1264, 2010.

7 Marek Cygan, Marcin Pilipczuk, Michał Pilipczuk, and Jakub Onufry Wojtaszczyk. A polynomial algorithm for 3-compatible coloring and the stubborn list partition problem (the stubborn problem is stubborn no more). SIAM Journal on Computing, 41(4):815-828, 2012.

8 Simone Dantas, Celina M. H. de Figueiredo, Sylvain Gravier, and Sulamita Klein. Finding $H$-partitions efficiently. RAIRO - Theoretical Informatics and Applications, 39(1):133-144, 2005.

9 Simone Dantas, Frédéric Maffray, and Ana Silva. $2 K_{2}$-partition of some classes of graphs. Discrete Applied Mathematics, 160(18):2662-2668, 2012.

10 Celina M. H. de Figueiredo. The P versus NP-complete dichotomy of some challenging problems in graph theory. Discrete Applied Mathematics, 160(18):2681-2693, 2012.

11 Yuri Faenza, Gianpaolo Oriolo, and Gautier Stauffer. Solving the weighted stable set problem in claw-free graphs via decomposition. Journal of the ACM, 61(4):20:1-20:41, 2014.

12 Tomás Feder and Pavol Hell. List homomorphisms to reflexive graphs. Journal of Combinatorial Theory, Series B, 72(2):236-250, 1998.

13 Tomás Feder, Pavol Hell, Sulamita Klein, and Rajeev Motwani. List partitions. SIAM Journal on Discrete Mathematics, 16(3):449-478, 2003.

14 Jirí Fiala, Marcin Kaminski, and Daniël Paulusma. A note on contracting claw-free graphs. Discrete Mathematics 83 Theoretical Computer Science, 15(2):223-232, 2013.

15 Herbert Fleischner, Egbert Mujuni, Daniël Paulusma, and Stefan Szeider. Covering graphs with few complete bipartite subgraphs. Theoretical Computer Science, 410(21-23):20452053, 2009.

16 Petr A. Golovach, Matthew Johnson, Barnaby Martin, Daniël Paulusma, and Anthony Stewart. Surjective $H$-colouring: New hardness results. Computability, to appear.

17 Frank Harary. Graph Theory. Reading MA, 1969. 
18 Pavol Hell. Graph partitions with prescribed patterns. European Journal of Combinatorics, 35:335-353, 2014.

19 Danny Hermelin, Matthias Mnich, Erik Jan van Leeuwen, and Gerhard J. Woeginger. Domination when the stars are out. CoRR, abs/1012.0012, 2010.

20 Danny Hermelin, Matthias Mnich, Erik Jan van Leeuwen, and Gerhard J. Woeginger. Domination when the stars are out. In Proc. ICALP 2011, volume 6755 of Lecture Notes in Computer Science, pages 462-473, 2011.

21 Takehiro Ito, Marcin Kaminski, Daniël Paulusma, and Dimitrios M. Thilikos. On disconnected cuts and separators. Discrete Applied Mathematics, 159(13):1345-1351, 2011.

22 Takehiro Ito, Marcin Kaminski, Daniël Paulusma, and Dimitrios M. Thilikos. Parameterizing cut sets in a graph by the number of their components. Theoretical Computer Science, 412(45):6340-6350, 2011.

23 Marcin Kaminski, Daniël Paulusma, Anthony Stewart, and Dimitrios M. Thilikos. Minimal disconnected cuts in planar graphs. Networks, 68(4):250-259, 2016.

24 Andrew D. King. Claw-free graphs and two conjectures on $\omega, \Delta$, and $\chi$. $\mathrm{PhD}$ thesis, University, Montreal, Canada, 2009.

25 Andrew D. King and Bruce A. Reed. Bounding $\chi$ in terms of $\omega$ and $\delta$ for quasi-line graphs. Journal of Graph Theory, 59:215-228, 2008.

26 Barnaby Martin and Daniël Paulusma. The computational complexity of disconnected cut and $2 K_{2}$-partition. Journal of Combinatorial Theory, Series B, 111:17-37, 2015 (conference version Proc. CP 2011, LNCS 6876, 561-575).

27 Ross M. McConnell. Linear-time recognition of circular-arc graphs. Algorithmica, 37(2):93147, 2003.

28 Rafael B. Teixeira, Simone Dantas, and Celina M. H. de Figueiredo. The external constraint 4 nonempty part sandwich problem. Discrete Applied Mathematics, 159(7):661-673, 2011.

29 Narayan Vikas. Computational complexity of compaction to reflexive cycles. SIAM Journal on Computing, 32(1):253-280, 2002.

30 Narayan Vikas. Algorithms for partition of some class of graphs under compaction and vertex-compaction. Algorithmica, 67(2):180-206, 2013 (conference version Proc. COCOON 2011, LNCS 6842, 319-330). 